$10-2019$

\title{
Synthesis and Secretome Release by Human Bone Marrow Mesenchymal Stem Cell Spheroids within Three-dimensional Collagen Hydrogels: Integrating Experiments and Modelling
}

Jyotsna Joshi

Cleveland State University

Mohammadreza Dehghan Abnavi

Cleveland State University

Ephandthis and additional works at: https://engagedscholarship.csuohio.edu/encbe_facpub

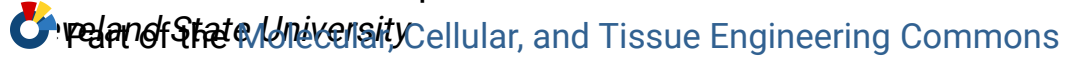

How does access to this work benefit you? Let us know!

\section{Publisher's Statement}

This is the accepted version of the following article: Joshi, J, Abnavi, MD, Kothapalli, CR. Synthesis and secretome release by human bone marrow mesenchymal stem cell spheroids within three-dimensional collagen hydrogels: Integrating experiments and modelling. J Tissue Eng Regen Med. 2019; 13, 1923- 1937. https://doi.org/10.1002/term.2943 , which has been published in final form at https://doi.org/10.1002/term.2943

\section{Repository Citation}

Joshi, Jyotsna; Abnavi, Mohammadreza Dehghan; and Kothapalli, Chandrasekhar R., "Synthesis and Secretome Release by Human Bone Marrow Mesenchymal Stem Cell Spheroids within Three-dimensional Collagen Hydrogels: Integrating Experiments and Modelling" (2019). Chemical \& Biomedical Engineering Faculty Publications. 164.

https://engagedscholarship.csuohio.edu/encbe_facpub/164

This Article is brought to you for free and open access by the Chemical \& Biomedical Engineering Department at EngagedScholarship@CSU. It has been accepted for inclusion in Chemical \& Biomedical Engineering Faculty Publications by an authorized administrator of EngagedScholarship@CSU. For more information, please contact library.es@csuohio.edu. 


\title{
Synthesis and secretome release by human bone marrow mesenchymal stem cell spheroids within three-dimensional collagen hydrogels: Integrating experiments and modelling
}

\author{
Jyotsna Joshi, Mohammadreza Dehghan Abnavi,Chandrasekhar R. Kothapalli
}

\begin{abstract}
Myocardial infarction results in loss of cardiac cell types, inflammation, extracellular matrix (ECM) degradation, and fibrotic scar. Transplantation of bone marrowderived mesenchymal stem cells (BM-MSCs) is being explored as they could differentiate into cardiomyocyte-like cells, integrate into host tissue, and enhance resident cell activity. The ability of these cells to restore lost ECM, remodel the inflammatory scar tissue, and repair the injured myocardium remains unexplored. We here elucidated the synthesis and deposition of ECM (e.g., elastin, sulfated glycosaminoglycans, hyaluronan, collagen type III, laminin, fibrillin, lysyl oxidase, and nitric oxide synthases), matrix metalloproteinases (MMPs) and their inhibitors (TIMPs), and other secretome (cytokines, chemokines, and growth factors) in adult human BM-MSC spheroid cultures within three-dimensional collagen gels. The roles of speciesspecific type I collagen and 5-azacytadine were assessed over a 28-day period. Results revealed that human collagen (but not rat-derived) suppressed MSC proliferation and survival, and MSCs synthesized and released a variety of ECM proteins and secretome over the 28 days. Matrix deposition is at least an order of magnitude lower than their release levels at every time point, most possibly due to elevated MMP levels and interleukins with a concomitant decrease in TIMPs. Matrix synthesis over the 28-day period was fitted to a competitive inhibition form of Michaelis-Menten kinetics, and the production and decay rates of ECM, MMPs, and TIMPs, along with the kinetic model parameters quantified. Such an integrated experimental and modelling approach would help elucidate the critical roles of various parameters (e.g., cell encapsulation and delivery vehicles) in stem cell-based transplantation therapies.

\section{KEYWORDS}

cardiomyocytes, competitive-inhibition model, matrix synthesis, mesenchymal stem cells, Michaelis-Menten kinetics, protein degradation, secretome release
\end{abstract}

\footnotetext{
Abbreviations:\$M-MSCs, bone marrow-derived mesenchymal stem cells; VSMCs, vascular smooth muscle cells; MI, myocardial infarction; sGAGs, sulfated glycosaminoglycans; LOX, lysyl oxidase; NO, nitric oxide; NOS, nitric oxide synthase; NOS1, neuronal nitric oxide synthase; iNOS, inducible nitric oxide synthase; eNOS, endothelial nitric oxide synthase; bFGF/FGF-2, basic fibroblast growth factor; EGF, epithelial growth factor; TGF- $\alpha$, transforming growth factor-alpha; TGF- $\beta$, transforming growth factor-beta; HGF, hepatocyte growth factor; IGF-1, insulin-like growth factor-1; VEGF, vascular endothelial growth factor; MMPs, matrix metalloproteases; TIMPs, tissue inhibitor of metalloproteinase; ILs, interleukins; M-CSF, macrophage colony stimulating factor; TNF- $a$, tumour necrosis factor-alpha; MCP-1, monocyte chemoattractant protein; MIP-1a, macrophage inflammatory protein; RANTES, regulated on activation, normal Tcell expressed and secreted; MCP-3 MCP-3/CCL7, monocyte-specific chemokine 3; IP-10, interferon- $\gamma$ inducible protein; TSG-6, TNFa stimulated gene/protein 6
} 


\section{1 | INTRODUCTION}

Adult mammalian myocardium comprises cardiomyocytes, fibroblasts, endothelial cells, smooth muscle cells, stem and progenitor cells, nerve endings, and blood cells, with cardiomyocytes alone occupying almost 75\% of myocardial volume (Bowers, Banerjee, \& Baudino, 2010; Kapelko, 2001). Myocardial tissue also houses matrix components that contribute to the maintenance of tissue functions during development, haemostasis, and disease (Lockhart, Wirrig, Phelps, \& Wessels, 2011). Myocardial cells uniquely contribute to the turnover of extracellular matrix (ECM), with cardiac fibroblasts producing collagens I and III, fibronectin, matrix metalloproteinases (MMPs), tissue inhibitors of MMPs (TIMPs), interleukins (ILs), TGF- $\beta$, TNF- $\alpha$, and endothelin, and cardiomyocytes contributing to collagens IV and VI, laminins, and proteoglycans (Bowers et al., 2010; Fan, Takawale, Lee, \& Kassiri, 2012). Collagens are the most abundant myocardial matrix components, with type I being the major myocardial structural protein constituting $75-80 \%$ of the total collagen (LeGrice, Pope, \& Smaill, 2005). Apart from type I, type III collagen constitutes 15-20\% and forms fine pericellular network, whereas minor amounts of other collagen types form basement membrane (e.g., collagen IV) or found in the cardiac interstitium with linkages to other collagens (collagens $\mathrm{V}$ and $\mathrm{VI}$; LeGrice et al., 2005). In addition to collagens, proteoglycans, glycoproteins, elastin, fibronectin, and laminin also play a major role in cell matrix organization (Bowers et al., 2010). Elastin is found in the walls of blood vessels and in the cardiac interstitium and plays a role in tissue organization, strength, and elasticity (Fomovsky, Thomopoulos, \& Holmes, 2010). Elastin biosynthesis involves release of soluble tropoelastin monomers that aggregate and mature on microfibrillar scaffolds composed mainly of fibrillin, with LOX mediating the crosslinking, stability, and assembly of tropoelastin monomers (Kielty, Sherratt, \& Shuttleworth, 2002).

Other glycoproteins (thrombospondins and tenascins), proteoglycans (versican and biglycan), and GAGs (hyaluronan, heparin, heparin sulfate, chondroitin sulfate, dermatan sulfate, and keratin sulfate) contribute to the nonstructural component of the myocardial matrix. GAGs and proteoglycans also aid in matrix hydration and tissue plasticity needed for cell migration, proliferation, and cardiac contractility (Pelouch, Dixon, Golfman, Beamish, \& Dhalla, 1993; Rienks, Papageorgiou, Frangogiannis, \& Heymans, 2014). Nitric oxide (NO) generated from NO synthase (NOS) plays crucial role in cardiac muscle contraction, and all three isoforms of NOS (NOS1, NOS2 [iNOS], and NOS3 [eNOS]) are present distinctly within cardiomyocytes (Förstermann \& Sessa, 2011). Thus, myocardial ECM is a dynamic and intricate assembly of multiple matrix components and a rich storehouse of bioactive molecules that essentially mediate cell signalling, matrix synthesis, and degradation processes via autocrine or paracrine mechanisms (Spinale, 2007).

In severe cases of myocardial infarction (MI), there could be loss of up to $50 \mathrm{~g}$ of cardiac tissue with a concomitant loss of millions of cardiomyocytes per gram of tissue (Zimmermann et al., 2006). To mitigate such loss, the cardiac intrinsic repair follows soon after cardiomyocyte death, but the outcome is usually adverse and varies with the extent of injury (Altara et al., 2016; Frangogiannis, 2014). Thus, cardiac tissue regeneration approaches utilizing stem cells, biomaterial scaffolds, and growth factors are being explored to replace the lost cell populations and restore the structure-function properties of the diseased tissue. Human bone marrow-derived mesenchymal stem cells (HBM-MSCs) are gaining attention in cardiac therapy due to their ability to release a wide repertoire of paracrine factors (termed secretome; Ranganath, Levy, Inamdar, \& Karp, 2012), which enhances survival and function of cardiomyocytes, enables activation, proliferation, and differentiation of endogenous cardiac progenitor cells, and inhibits tissue fibrosis (Cashman, Gouon-Evans, \& Costa, 2013; Williams \& Hare, 2011). We had earlier reported that a combination of rat tail-derived type I collagen, 5-azacytidine, and spheroid formation provides an optimal 3D milieu for the evolution of cardiomyocyte-like cells from HBM-MSCs (Joshi, Mahajan, \& Kothapalli, 2018). However, ECM protein expression and synthesis and matrix remodelling capabilities of these cells remain relatively unknown, which is critical for their applications in situ post-MI.

This study is based on the hypothesis that HBM-MSC survival, proliferation, ECM synthesis, and secretome release is regulated by 3D scaffold source (rat vs. human collagen), aza exposure (10 $\mu \mathrm{M})$, and culture duration (over 28 days). This study is also partially driven by immune concerns over the utility of animal-derived collagen gels for clinical applications (Gorgieva \& Kokol, 2011). Finally, the timedependent evolution of protein synthesis and degradation (i.e., remodelling) within these cultures, mediated by MMPs (enzyme) and TIMPs (inhibitor), was fitted to a competitive inhibition form of MichaelisMenten reaction kinetics and the model parameters elucidated.

\section{2 | MATERIALS AND METHODS}

\section{1 | Preparation of collagen hydrogels}

Type 1 rat collagen extracted and purified from rat tail tendon (Corning $®$ ) and type 1 human collagen extracted and purified from neonatal foreskin derived-fibroblast cultures (Vitrocol®); Advanced Biomatrix) were used to form $2 \mathrm{mg} / \mathrm{mL}$ gels, by adjusting the amounts of stock collagen, $\mathrm{NaOH}, 10 \times$ PBS, and culture media, as per vendor's protocol.

\section{2 | Human BM-MSC culture and spheroid formation}

Cryopreserved Poietics ${ }^{\mathrm{TM}}$ normal HBM-MSCs (PT-2501), isolated from adult human bone marrow of the posterior iliac crests, were obtained from Lonza (Walkersville, MD, USA). The cells were cryopreserved after the second passage and had $\geq 90 \%$ of CD105, CD166, CD90, CD44, and CD73. HBM-MSC spheroids were formed using a hanging drop method, from three to six passage cells, using Gibco $^{\text {TM }}$ DMEM. In brief, loose cells were trypsinized, neutralized, centrifuged at $500 \mathrm{rcf}$ for $5 \mathrm{~min}$, and suspended in DMEM culture media. Cell suspensions $(\sim 1,000$ cells $/ 20 \mu \mathrm{l})$ were pipetted into the lids of Petri plates that 
were inverted and placed over Petri dishes containing PBS, followed by a $24-\mathrm{hr}$ incubation under standard aseptic culture conditions. After 1 day of incubation, hanging drops were gently transferred on tissue culture plastic (TCP) or sandwiched within rat or human collagen hydrogels. Cell-loaded hydrogels were incubated under standard culture conditions to induce their gelation prior to adding culture media supplemented with and without $10 \mu \mathrm{M}$ aza. Rat collagen took 30 min, whereas human collagen took 90 min to gel, matching the vendor's specifications. Media was changed in all cultures every $48 \mathrm{hr}$ over the 28-day period, including replenishing fresh aza. TCP cultures did not receive aza and act as a baseline for each quantified measure and for comparing with 3D cultures.

\section{3 | Biochemical assays for matrix quantification}

Proteins released into the pooled media and those deposited in the cell layers at Days 1, 12, and 28 of cultures were quantified in all test conditions. The pooled spent culture media was collected and stored at $-20^{\circ} \mathrm{C}$, transferred to Eppendorf safe-lock tubes, and centrifuged at $14,000 \mathrm{rpm}$ for $15 \mathrm{~min}$ at $4^{\circ} \mathrm{C}$. The supernatant was discarded, and the pellet homogenously suspended in distilled deionized water for biochemical analyses. The deposited matrix proteins within the cell layers were harvested from the cultures at various time points. The cultures were first rinsed with $1 \times$ PBS, trypsinized for cell-laden gel detachment from the plates, neutralized with culture media, pipetted into Eppendorf safe-lock tubes, and centrifuged at 500 rcf for 5 min. The supernatant was discarded, and the cell pellet was homogenously suspended in distilled deionized water and stored at $-20^{\circ} \mathrm{C}$ for biochemical analyses.

\subsection{1 | DNA assay}

The total double-stranded DNA was quantified using DNA quantification kit (Sigma-Aldrich), which is based on a fluorescent dye bisbenzimide $\mathrm{H} 33258$ (Hoechst 33258). Samples were excited at $360 \mathrm{~nm}$, and the fluorescence emissions at $460 \mathrm{~nm}$ were recorded using a microplate reader (Biotek Synergy $\mathrm{H} 1 \mathrm{~m}$ ). A calibration curve was prepared using DNA concentration of the standards provided with the kit versus their respective fluorescence intensities. The least squares regression equation was fitted for this curve, which was used to determine the DNA amounts in the samples. Total cell density in each sample was calculated assuming 8.5 pg DNA per cell (Wang, Tran, Seshareddy, Weiss, \& Detamore, 2009).

\subsection{2 | BCA assay}

Total protein released into pooled media and deposited within cell cultures was quantified using Pierce ${ }^{\mathrm{TM}}$ BCA protein assay kit. The absorbance in processed samples was read at $562 \mathrm{nM}$ using a microplate reader, and the total protein in samples was determined using the least squares regression equation from standard curve generated from BSA standards provided in the kit. Total protein in cell-free hydrogels was determined to account for the baseline of protein amounts within cell pellet samples in respective hydrogels.

\subsection{3 | sGAG assay}

The total sGAGs deposited within the cell layers and that released in the pooled media were quantified using a quantitative dye-binding sGAG assay (Kamiya Biomedical Company), and the processed samples were read at $610 \mathrm{~nm}$ using a microplate reader. A standard curve generated from the sGAG samples provided with the kit was used to calculate sGAG amounts in each test sample.

\subsubsection{HA assay}

The hyaluronic acid amounts deposited within cell layers and in the pooled media were quantified using a quantitative enzyme-linked immunoassay (HA-ELISA assay kit, Echelon, Salt Lake City, UT). Absorbance was measured at $403 \mathrm{~nm}$ using a microplate reader, and the standard curve generated was used to find HA levels in the test samples.

\subsection{5 | Fastin assay for elastin protein}

The total elastin deposited within the cell layers (i.e., matrix elastin) and that released in the pooled media (i.e., tropoelastin) was quantified using a Fastin ${ }^{\mathrm{TM}}$ elastin assay (Accurate Chemical and Scientific Corp; Westbury, NY). The assay is based on the principle that the dye reagent, TPPS (5,10,15,20-teraphenyl-21H,23H-porphyrine), has an affinity to bind with solubilized elastin. Absorbance was measured using the microplate reader at $513 \mathrm{~nm}$, and a standard curve was generated to quantitate elastin in the samples.

\subsection{6 | LOX functional activity}

LOX activity in the cell matrix and in the pooled media was determined using a sensitive fluorescence-based assay (Amplex ${ }^{\circledR}$ Red Hydrogen Peroxide/Peroxidase assay kit, Molecular Probes) that uses the Amplex Red reagent (10-acetyl-3,7-dihydroxyphenoxazine) to detect $\mathrm{H}_{2} \mathrm{O}_{2}$. This assay is based on the principle that LOX amount is proportional to the released $\mathrm{H}_{2} \mathrm{O}_{2}$ because LOX oxidatively deaminates alkyl monoamines and diamines (Palamakumbura \& Trackman, 2002; Simmers, Gishto, Vyavahare, \& Kothapalli, 2015). Absorbance was read at $560 \mathrm{~nm}$, and a linear standard curve was derived to find $\mathrm{H}_{2} \mathrm{O}_{2}$ /LOX in the samples.

\section{4 | Secretome release}

The cytokine/chemokine/growth factor cocktail pertinent to cardiomyocyte differentiation and cardiac tissue regeneration was quantified using Discovery Assays ${ }^{\circledR}$ (Eve Technologies, Alberta, Canada) according to the manufacturer's protocol. Briefly, the collected pooled media from cell cultures was thawed on ice, centrifuged, and required volumes aliquoted into $0.5-\mathrm{ml}$ polypro snap-cap tubes. Nine essential 
metalloproteases and their inhibitors (MMPs and TIMPs, respectively) were quantified using human MMP and TIMP panel assay (assay sensitivity: 0.5-14 pg/ml); 42 essential cytokines/chemokines quantified using human cytokine array/chemokine array assay (assay sensitivity: $0.5-10 \mathrm{pg} / \mathrm{ml}$ ); and TGF $\beta-1,2$, and 3 quantified using TGF- $\beta$ array (assay sensitivity: $2-6 \mathrm{pg} / \mathrm{ml}$ ). These assays utilize multiplexing LASER bead technology where fluorophore beads provide a platform for multiple cytokine and chemokine detection from a single assay using the bead analyser (Bio-Plex 200).

\subsection{IF labelling and imaging}

Primary antibodies (Santa Cruz Biotechnology) for collagen type III, laminin, fibrillin, NOS2, and NOS3 deposited by the MSC spheroids were assessed in 28-day culture conditions. Cell-laden hydrogels were fixed and stained with antibodies and DAPI at the end of each culture period (Joshi et al., 2018). Live/Dead ${ }^{\circledR}$ Viability/Cytotoxicity Kit (Molecular Probes) was used to determine the cell viability and toxicity at various culture conditions (Gishto, Farrell, \& Kothapalli, 2015). All fluorescence images were acquired using Zeiss Axiovert A1 florescence microscope equipped with Hamamatsu camera and an image acquisition software. Multiple images from at least three independent wells were analysed for each case.

\subsection{Western blots}

SDS/PAGE western blot analysis was performed for semiquantitative analysis of collagen type III, laminin, fibrillin, NOS2, and NOS3 proteins (Simmers et al., 2015). Blots were performed for both the released proteins in pooled media and that deposited within the cell layers, using samples specified in Section 2.3. Band intensities were normalized to total protein amounts (BCA assay) and further to cell densities within respective culture conditions.

\subsection{Statistical analysis}

All data were represented as mean \pm standard error of $n \geq 3$ wells per condition, with at least three independent replicates. Statistical analysis was performed using GraphPad Prism 5 for Student's $t$ test, oneway analysis of variance (ANOVA) followed by Tukey's test, or twoway ANOVA followed by Bonferroni post hoc test, to find the statistical significance and determine the pairs of groups that were significantly different $(p<.05)$.

\section{3 | RESULTS}

\subsection{Cell viability in rat and human collagen gels}

Cell viability within rat collagen cultures were $\geq 80 \%$ in all culture conditions and were not influenced by aza treatment (Figure 1). On the contrary, viability ranged between $50 \%$ and $60 \%$ in human collagen cultures and was significantly lower compared with that in rat collagen cultures at all corresponding time points $(p<.05)$. However, aza presence did not impact cell viability in human collagen cultures.

\subsection{Cell proliferation and matrix synthesis in 2D cultures}

The average cell density increased from 3,100 to $7,800(p<.05$; Figure 2a), with a simultaneous increase in average normalized total matrix protein deposition ( $\sim 6,300 \mathrm{pg}$ to $\sim 26 \mathrm{ng} ; p=.0002$; Figure 2 c), from Days 12 to 28 . Although average normalized total protein release in media increased from Days 1 to $12(\sim 280$ to $990 \mathrm{ng} ; p<.05$; Figure 2d), it decreased from Days 12 to 28 ( 990 to $380 \mathrm{ng}, p=$ .0029). The normalized LOX deposition increased from Days 1 to 12 $(\sim 150$ to $270 \mathrm{pM})$ and lowered by Day 28 ( 100 pM, $p<.05)$; similarly, normalized LOX activity in pooled media increased from Days 1 to 12 ( 8 to $11 \mathrm{nM} ; p<.05)$ but reduced from Days 12 to 28 ( 11 to $4.7 \mathrm{nM}$; $p<.0001$; Figure 2b). Although normalized sGAG and elastin matrix deposition slightly increased from Days 1 to 12 , it reduced from Days 12 to 28 ( $\sim 365$ and 280 pg to $\sim 30$ and 90 pg; $p=.01$; Figure $2 c$ ). On the other hand, normalized sGAG and tropoelastin release into media increased from Days 1 to 12 ( 650 and 880 pg to $~ 3,300$ and 2,700 $\mathrm{pg}$, respectively; $p=.004$; Figure $2 \mathrm{~d}$ ) and plateaued from Days 12 to 28. Normalized HA in matrix reduced from Days 1 to $12(\sim 36$ to 18 pg; $p<.001$; Figure 2c) and from Days 12 to $28(\sim 18$ to 7 pg; $p<$ .0001); however, HA release into pooled media increased with culture duration from Days 1 to 12 ( 38 to 65 pg; $p=.0225$; Figure $2 d$ ). In general, levels of total protein, LOX, elastin, and GAGs were orders of magnitude higher in pooled media compared with that deposited as matrix. Moreover, elastin and sGAG levels were similar in matrix or in pooled media and orders of magnitude higher than HA levels. These values establish the baseline for various ECM proteins synthesized and released within 2D MSC cultures in the absence of exogenous aza.

\subsection{MSC proliferation, protein release, and deposition within rat collagen gels}

Within rat collagen, the average cell density in controls ( $0 \mu \mathrm{M}$ aza) increased steadily with culture duration $(\sim 3,000,7,500$, and 1,700 for Days 1, 12, and 28, respectively; $p=.04$; Figure 2e). Normalized LOX deposition in matrix increased in controls over time (from $\sim 150$ to $400 \mathrm{pM} ; p=.02$ for Day 1 vs. Day 28; Figure 2f), although LOX release in pooled media steadily reduced with culture duration $(\sim 7,900$ to $1,900 \mathrm{pM} ; p<.05)$. Normalized total protein and sGAG deposition in controls increased from Days 1 to 12 but decreased by Day 28 ( 55 ng and 325 pg on Day 12 to $\sim 12$ ng and 83 pg on Day 28 , respectively; $p<.001$; Figure $2 \mathrm{~g}$ ), whereas the total protein and sGAG release into media stayed steady throughout the culture period (Figure $2 \mathrm{~h}$ ). HA deposition in matrix and release into pooled media, on a per cell basis, lowered by Day 12 ( 8 and $20 \mathrm{pg}$ ) and Day 28 ( 5 and $16 \mathrm{pg})$ in control cultures, compared with Day 1 ( 36 and $38 \mathrm{pg} ; p<$ .001 in both cases). Although normalized elastin matrix deposition reduced in controls from Day $1(\sim 140 \mathrm{pg})$ to Day $12(\sim 65 \mathrm{pg})$ and from 


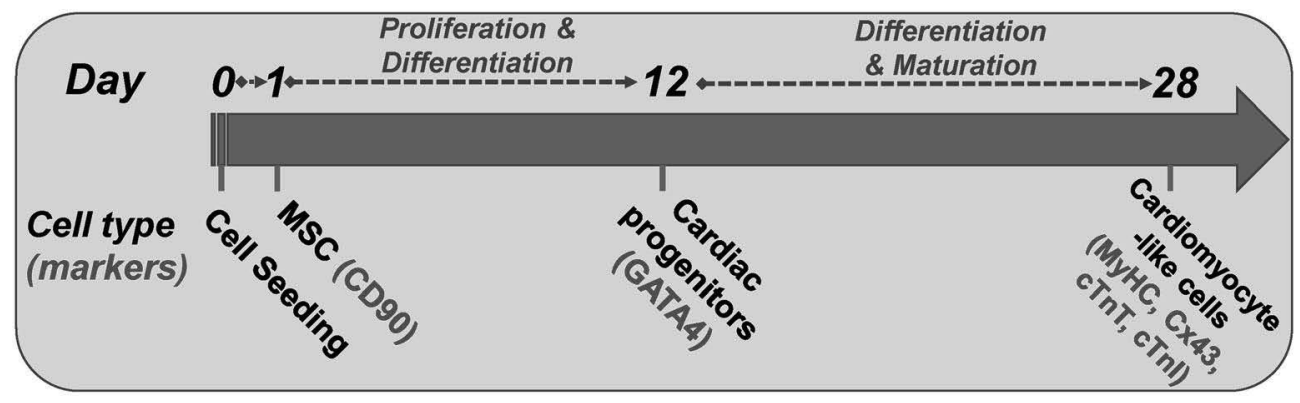

(a)

(a) Day 1

Day 12

Day 28

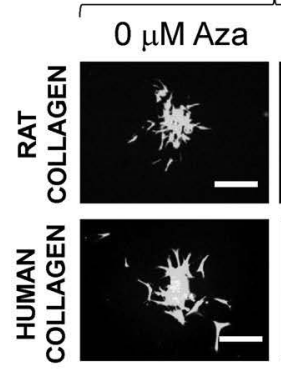

(b)
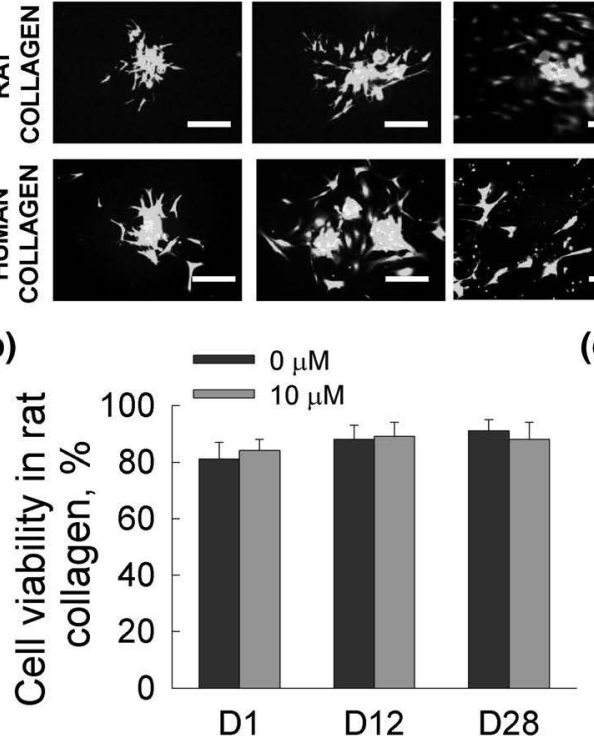

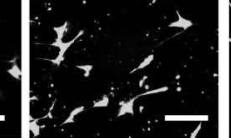

(c)

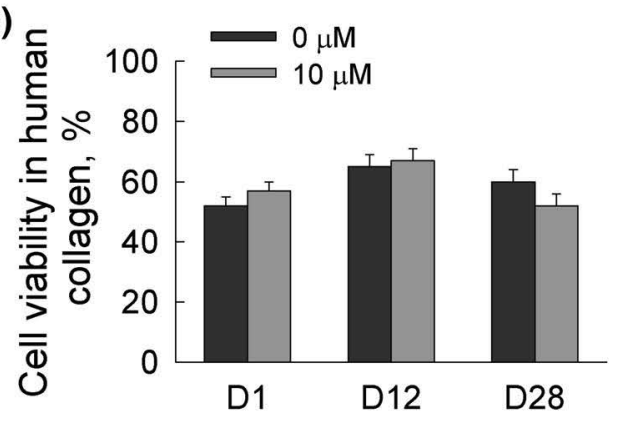

FIGURE 1 Schematic of the MSC evolution in 2D and 3D cultures over a 28-day culture period (Joshi et al., 2018): cell spheroids seeding on Day 0 , undifferentiated MSC identification on Day 1, MSC differentiation to cardiac progenitor cells on Day 12, and mature cardiomyocytes formation by Day 28. Respective cell markers used at each time point were also shown. (a) Representative immunofluorescence images from LIVE/DEAD ${ }^{8}$ staining within rat or human collagen gels, on Days 1, 12, and 28, in the presence or absence of $10 \mu \mathrm{M}$ aza. Scale bar: $50 \mu \mathrm{m}$. Quantification (mean \pm standard error) of the cell viability from these images in rat (b) and human (c) type I collagen gels [Colour figure can be viewed at

wileyonlinelibrary.com]

Days 12 to 28 (undetectable; $p<.0001$; Figure $2 \mathrm{~g}$ ), tropoelastin release increased steadily over the culture duration $(p=.001$ for D12 [ 1,000 pg] vs. D28 [ 1,500 pg]; Figure 2h).

The overall average cell density in aza-treated cultures increased from Days 1 to 28 ( $p<.1$ for D1 with 3,400 vs. D28 with 10,000; Figure 2e). Normalized total protein matrix deposition in aza-treated samples (Figure 2g) increased from Days 1 to 12 ( 6 to $14 \mathrm{ng} ; p<$ $.05)$ and furthermore from Days 12 to 28 ( 14 to $50 \mathrm{ng} ; p<.0001)$. The normalized total protein release in pooled media reduced from Days 1 to 12 ( 275 to $117 \mathrm{ng} ; p<.01$; Figure $2 \mathrm{~h}$ ) but increased from Days 12 to $28(\sim 117$ to 480 ng; $p<.001)$. Normalized sGAG deposition in aza-treated cultures reduced with culture duration (Figure 2 g), although sGAG release into pooled media remained constant over the culture duration (Figure $2 \mathrm{~h}$ ). Similarly, an overall reduction in HA deposition as matrix and release into pooled media was noted in aza-treated cultures over the 28-day period (Figure 2g,h). Although elastin matrix amounts decreased with culture duration in aza-treated samples ( 140 pg on Day 1 to 25 pg on Days 12 and 28; $p<.05$; Figure $2 \mathrm{~g})$, tropoelastin release improved with culture duration $(p<.0001$ for D1 [ 850 pg] vs. D28 [3,000 pg]; Figure 2h). Finally, normalized LOX deposition in matrix steadily increased with culture duration, with significantly higher amounts on Day $28(\sim 1,200$ pM) versus Days 1 $(\sim 150 \mathrm{pM})$ and 12 ( 270 pM; $p=.001$; Figure 2f); however, LOX release into pooled media reduced over the 28-day period $(\sim 8,000$ pM on Day 1 vs. 3,200 pM on Day 28; $p<.05$; Figure 2f). In general, (a) the total protein, LOX, elastin, and GAG amounts in pooled media were much higher than that in matrix, in both controls and aza cultures, (b) aza-receiving cultures had lower levels of ECM proteins in both matrix and media at Day 12 (vs. controls), but higher levels by Day 28 , and (c) elastin and sGAG levels were comparable in all the 


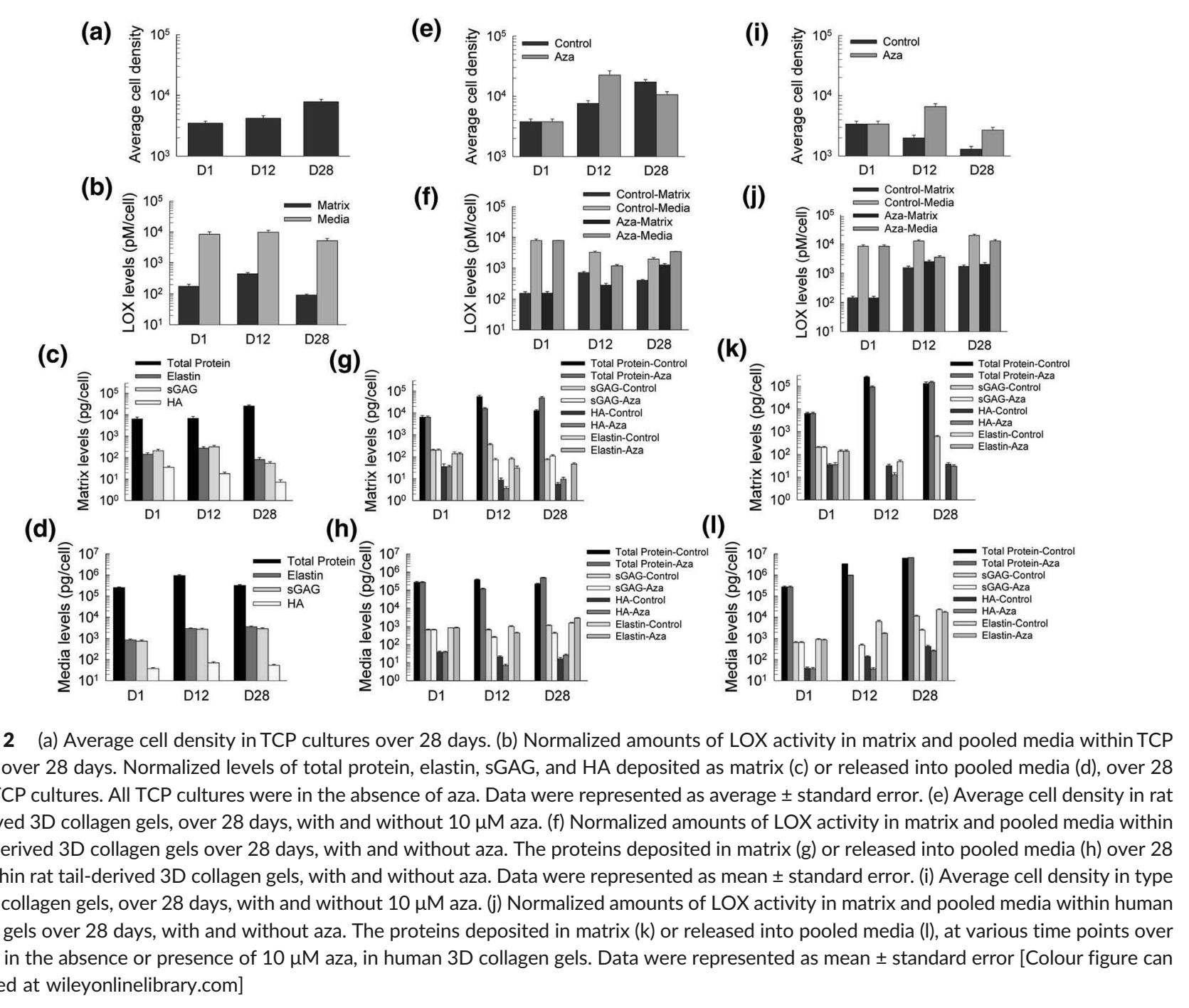

FIGURE 2 (a) Average cell density in TCP cultures over 28 days. (b) Normalized amounts of LOX activity in matrix and pooled media within TCP cultures over 28 days. Normalized levels of total protein, elastin, sGAG, and HA deposited as matrix (c) or released into pooled media (d), over 28 days in TCP cultures. All TCP cultures were in the absence of aza. Data were represented as average \pm standard error. (e) Average cell density in rat tail-derived 3D collagen gels, over 28 days, with and without $10 \mu \mathrm{M}$ aza. (f) Normalized amounts of LOX activity in matrix and pooled media within rat tail-derived 3D collagen gels over 28 days, with and without aza. The proteins deposited in matrix (g) or released into pooled media ( $\mathrm{h}$ ) over 28 days within rat tail-derived 3D collagen gels, with and without aza. Data were represented as mean \pm standard error. (i) Average cell density in type I human collagen gels, over 28 days, with and without $10 \mu \mathrm{M}$ aza. (j) Normalized amounts of LOX activity in matrix and pooled media within human collagen gels over 28 days, with and without aza. The proteins deposited in matrix $(\mathrm{k})$ or released into pooled media (l), at various time points over 28 days, in the absence or presence of $10 \mu \mathrm{M}$ aza, in human 3D collagen gels. Data were represented as mean \pm standard error [Colour figure can be viewed at wileyonlinelibrary.com]

culture conditions at all time points, and significantly higher than corresponding HA amounts.

\subsection{MSC proliferation, ECM release, and deposition within human collagen gels}

The average cell density decreased with culture duration in controls ( $3,000,2,000$, and 1,500 on Days 1,12 , and 28 , respectively); however, the differences were not statistically significant (Figure 2i). Compared with Day 1 levels, overall LOX amounts improved with culture duration, in both matrix and pooled media ( $p=.04$ and $p=.001$, respectively; Figure $2 \mathrm{j})$. The normalized total protein deposition as matrix increased in controls from Day 1 to Day $12(\sim 6.3$ to $250 \mathrm{ng}$; $p<.001$; Figure 2k) but reduced from Day 12 to Day 28 ( 250 to $100 \mathrm{ng} ; p<.05)$. Normalized total protein release in controls increased progressively from Day 1 to Day 28 ( 270 ng to $6 \mu \mathrm{g} ; p=.0062$; Figure 2l). sGAG deposition and release in control cultures was only detectable at Day 28 (Figure 2k,l). Although HA deposition as matrix did not significantly change with culture duration in controls, its presence in pooled media progressively increased over the 28 days ( 40 pg on Day 1 to 420 pg on Day 28; $p=.0003$; Figure 21). Normalized elastin deposition as matrix steadily and significantly decreased with culture duration in controls ( 140 pg on Day 1 and undetectable on Day 28; $p=.0004$; Figure $2 \mathrm{k}$ ), but elastin release progressively increased with culture duration ( 900 pg on Day 1, 6,500 pg by Day 12 , and 23 ng by Day 28; $p<.0001$; Figure 2l).

Average cell density in aza-treated cultures increased initially $(p<$ .05 for D1 with $\sim 3,000$ vs. D12 with $\sim 6,700$ ) but reduced to Day 1 levels by Day 28 ( $p<.001$ for D12 vs. D28) within human collagen gels (Figure 2i). Normalized total protein deposition and release into pooled media within aza cultures increased progressively during the culture period ( $p<.001$ in all cases; Figure $2 k, 1)$. Normalized sGAG amounts were undetectable in matrix at Days 12 and 28 within aza cultures but increased in pooled media from Day 12 to Day 28 (470 to 2,500 pg; $p$.001; Figure 2l). HA deposition in matrix remained unchanged by Day 28 ( $p>.05$ for D1 vs. D28; Figure 2k), whereas its release in pooled media increased multifold $(p<.0001$ for D1 with $\sim 40$ pg vs. D28 with 256 pg) within aza cultures. Normalized elastin deposition dropped in aza-treated samples with culture duration $(p<$ 
.0001 for D1 [140 pg] vs. D28 [undetectable]), whereas elastin release increased with culture duration ( $p<.0001$ for D1 [ 870 pg] vs. D28 [16.5 ng]; Figure 2l). LOX deposition and release increased with culture duration till Day 12 ( $p=.0445$ and $p=.0003$, respectively) in aza cultures, after which it plateaued (Figure 2j). Taken together, (a) the total protein, LOX, elastin, and GAG amounts in pooled media were much higher than that in matrix, in both controls and aza cultures, (b) aza-receiving cultures had lower levels of ECM proteins in both matrix and media at Day 12 (vs. controls), but similar levels by Day 28 (vs. controls), and (c) elastin and sGAG levels were comparable in all the culture conditions at all time points, and significantly higher than corresponding HA amounts.

\subsection{Immunofluorescence labelling and western blot analysis of ECM proteins}

The presence of type III collagen, laminin, fibrillin, NOS2, and NOS3 in 2D and 3D controls and aza-receiving cultures, at various time points, was qualitatively visualized from immunofluorescence images (Figure 3) and quantified from western blot analysis (Figure 4). The western blot band intensities were first normalized to total protein amounts (BCA assay) and further to cell densities within respective cultures. All five proteins were detectable in cell matrix within rat or human collagen gels from Day 12, with none detectable in Day 1 cultures from images and western blots. Human collagen gels (both

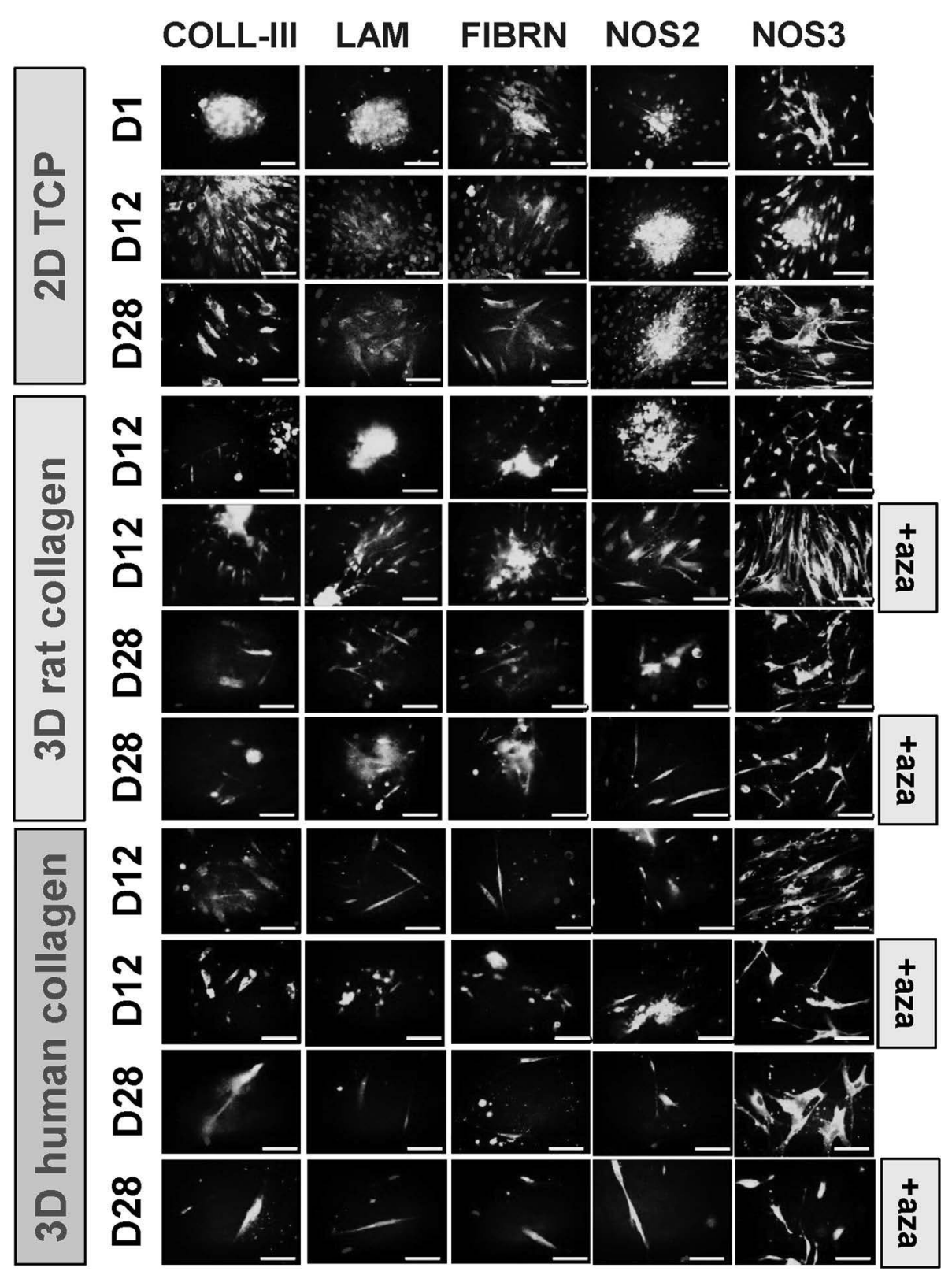

FIGURE 3 Representative immunofluorescence images of cardiac extracellular matrix proteins (collagen type III, laminin, and fibrillin) and nitric oxide synthases (NOS2 and NOS3) in cultures within 2D TCP and 3D rat or human collagen gels, at various time points over 28 days, in the presence or absence of $10 \mu \mathrm{M}$ aza. Scale: $100 \mu \mathrm{m}$ [Colour figure can be viewed at wileyonlinelibrary.com] 


\section{(a) Cell matrix}
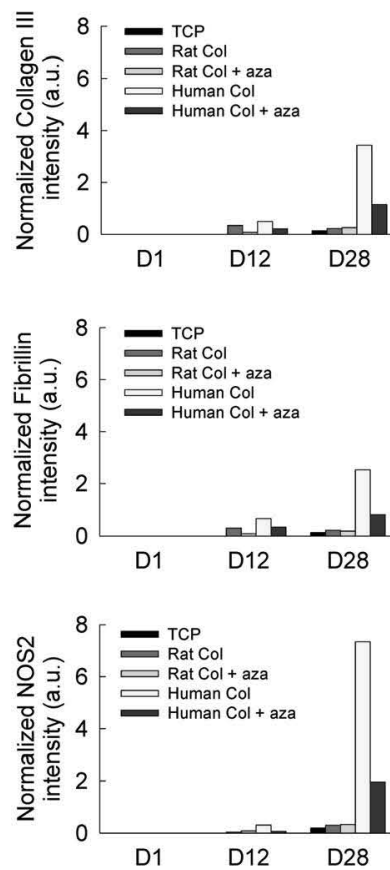

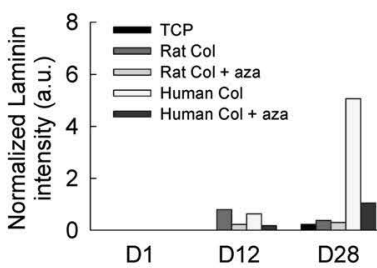

\section{(b) Pooled media}
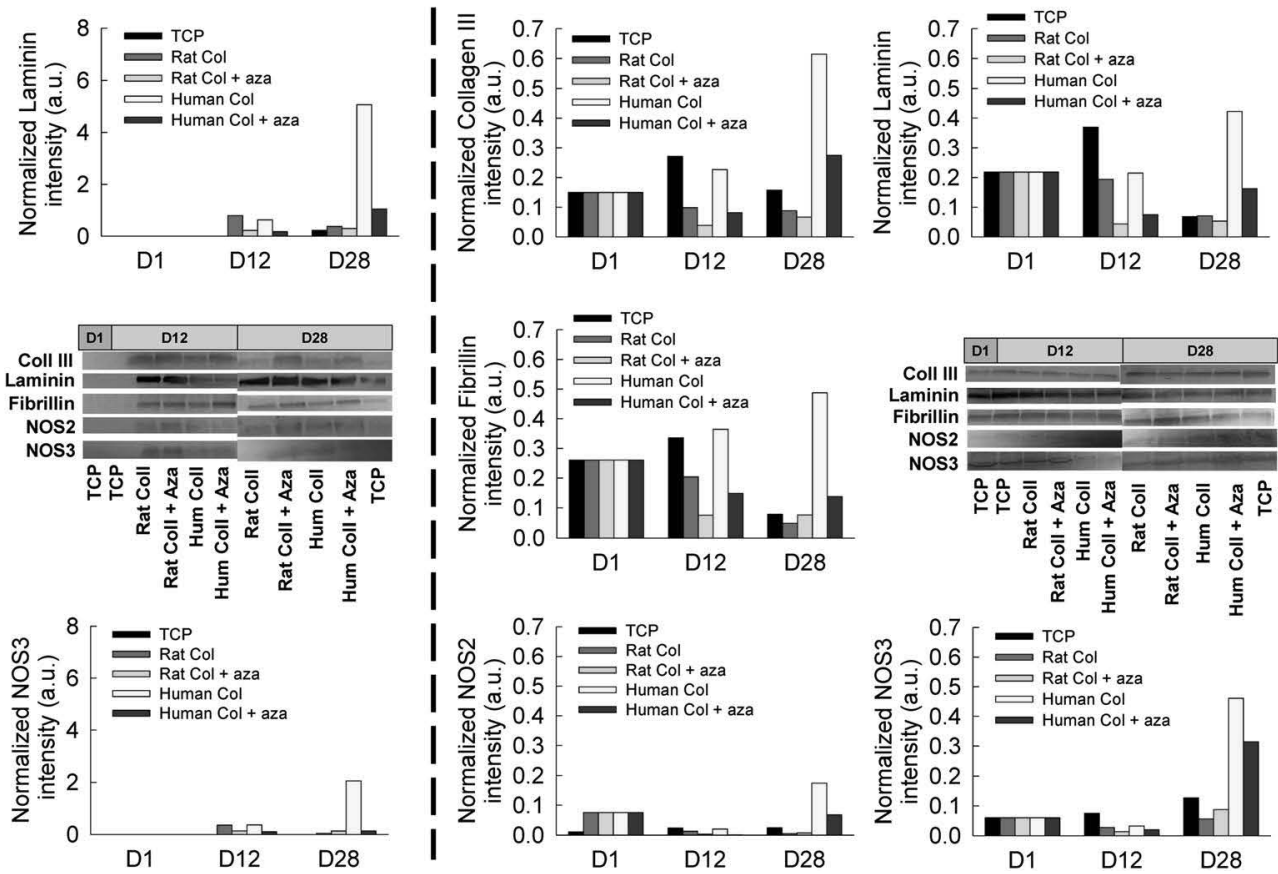

FIGURE 4 Quantification of bands from western blot analysis of ECM proteins (collagen type III, laminin, and fibrillin) and nitric oxide synthases (NOS2 and NOS3), isolated from (a) cell matrix and (b) pooled media. The intensity from western blot bands was normalized to the intensity of total protein expressed and further to the cell count in respective cases. Representative gel bands were shown for each protein at respective time points (D1, D12, and D28) [Colour figure can be viewed at wileyonlinelibrary.com]

Tissue Culture Plastic

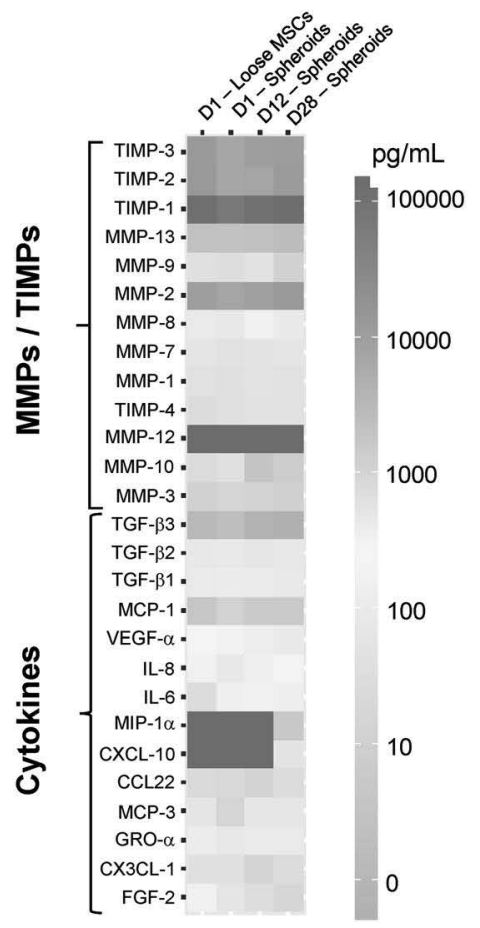

\section{Human Collagen type I}

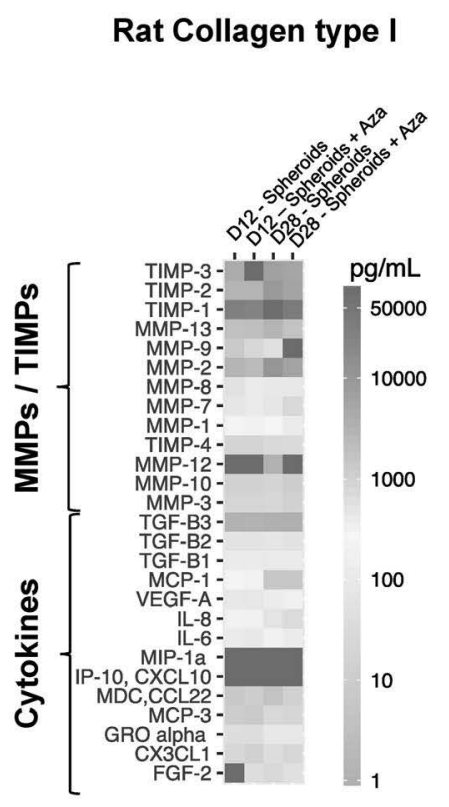

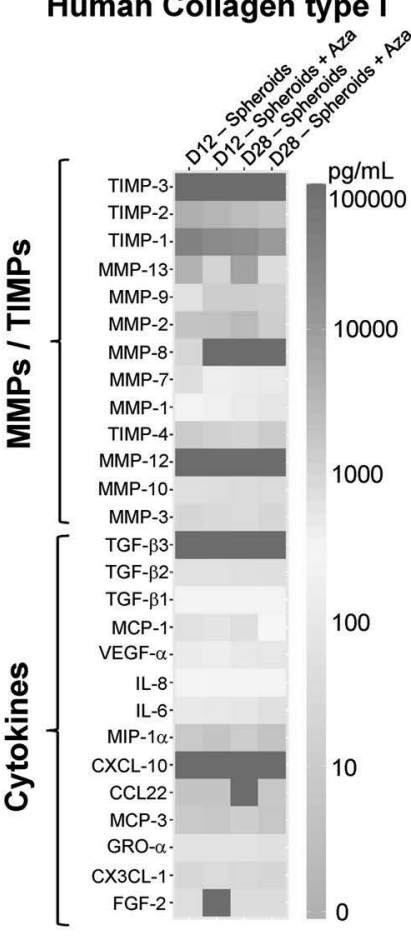

FIGURE 5 Heat maps showing the amounts of various MMPs, TIMPs, and cytokines quantified using LASER bead technology (Eve Technology) within (a) TCP, (b) 3D type I rat collagen cultures, and (c) 3D type I human collagen cultures, under different conditions over 28 days [Colour figure can be viewed at wileyonlinelibrary.com] 
controls and aza-exposed) promoted deposition of collagen type III, NOS2, fibrillin, and laminin within matrix layers (Figure 4a), by Day 28 , compared with other gel types. The presence of aza did not promote significant deposition of any of these proteins compared with control cultures, in both human and rat collagen gels. Qualitatively, the percentage of cells that stained positive for NOS3 in all culture conditions was significantly higher compared with those for other proteins (Figure 3). Interestingly, western blot analysis of these proteins from pooled media aliquots (Figure $4 b$ ) suggested that collagen type III, laminin, and fibrillin release improved by Day 12 in only TCP and human collagen gel cultures, and the amounts of these proteins either remained stable or reduced by Day 28 in most of the culture conditions except in human collagen gels.

\subsection{Secretome analysis}

Out of the 42 cytokines quantified (Figure 5; Data S1), eotaxin-1 and IL-15 were not expressed at all; EGF, TGF- $a$, FIt-3L, GM-CSF, IFN- $\alpha 2$, IL-10, IL-12 (p40), IL-12 (p70), PDGF-AA, IL-13, PDGF-BB, CD154, IL-17A, IL-1RA, IL-1a, IL-1 $\beta$, IL-2, IL-3, IL-5, IL-7, MIP-1B, RANTES, TNF- $\alpha$, TNF- $\beta$, IL18, and IL- 4 were the least expressed $(\leq 5 \mathrm{pg} / \mathrm{ml})$; FGF-2 (bFGF), Fractalkine/CX3CL1, GRO-a, MCP-3/CCL7, CCL22, IP-10/CXCL10, and MIP-1a were moderately expressed $(\leq 100$ $\mathrm{pg} / \mathrm{ml}) ; \mathrm{IL}-6, \mathrm{IL}-8$, and VEGF-a were highly expressed (100-600 $\mathrm{pg} / \mathrm{ml})$; and MCP-1 was profusely expressed (<2,000 pg/ml). Similarly, of the three cytokines quantified using TGF- $\beta$-Plex Discovery Assay, TGF- $\beta 1$ was highly expressed (350-450 pg/ml), TGF- $\beta 2$ was moderately expressed $(\leq 100 \mathrm{pg} / \mathrm{ml})$, and TGF- $\beta 3$ was the least expressed $(\leq 2 \mathrm{pg} / \mathrm{ml})$ in all culture conditions. Finally, of the 13 cytokines quantified using MMP 9-Plex and TIMP 4-Plex Discovery Assays, MMPs 1, 3 , $7,8,9,10$, and 12 were mostly within $0-200 \mathrm{pg} / \mathrm{ml}$ range, MMPs 2 and 13 were within $1,000-12,000 \mathrm{pg} / \mathrm{ml}$ range, TIMP-4 was less than 50 $\mathrm{pg} / \mathrm{ml}$, and TIMPs 1,2 , and 3 were in a broad range $(0-80,000 \mathrm{pg} / \mathrm{ml})$.

We further interpreted the release of these cytokines based on culture conditions as well as categorized into growth factors, chemokines, and proteases/inhibitors. TGF- $\beta 1, \beta 2$, and $\beta 3$ levels did not vary significantly with culture conditions. VEGF- $\alpha$ amount was the highest in loose MSCs that reduced in spheroids on TCP by Day 28 but increased upon aza exposure in 3D rat or human collagen cultures. Fibrotic cytokine FGF-2 amount was also the highest in loose MSCs on TCP, followed by spheroids in TCP, but lower within 3D collagen cultures (Figure 5). Potent inflammatory cytokine MCP-1 levels drastically reduced in rat and human collagen gels by Day 12 but increased within rat collagen by Day 28 in the presence or absence of aza. However, MCP-1 amount in human collagen cultures stayed the same from Day 12 to Day 28 and was further reduced by azatreatment. IL-6 and MCP-1 levels significantly reduced within rat collagen by Day 12, but increased by Day 28; however, their levels reduced by Day 28 within human collagen cultures. IL-8 release drastically increased by Day 28 within rat collagen cultures compared with $2 \mathrm{D}$ or human collagen cultures, and aza further enhanced these levels. IP-10 (CXCL10) was not expressed in any culture, except by spheroids cultured on TCP for 28 days. MIP-1a was not expressed within rat collagen gels but was faintly released in TCP and human collagen gels. Finally, CX3CL1 and MDC (CCL22) were not significantly influenced by scaffold type, aza exposure, or culture duration. These results suggest that spheroid formation, 3D collagen gels, collagen source (human vs. rat), and aza strongly influenced the release of MCP-1, VEGF, IL-6, IL-8, and FGF-2.

The basal MMP-1 levels in TCP were increased in 3D collagen (rat or human), although the amounts were lowered by more than half with aza-treatment. MMP-2 was profusely expressed in all cultures, with the highest in TCP and the lowest within human collagen cultures. Interestingly, within rat collagen gels, MMP-2 levels reduced by Day 12 (with or without aza) but increased by Day 28. MMP-3 levels remained low in all cultures, MMP-7 levels varied based on substrate type (TCP < rat collagen gel < human collagen gel), whereas MMP-8 levels were the least in human collagen gels followed by rat collagen gels and TCP. Although MMP-9 levels were low in all cultures, they significantly varied depending on culture conditions and duration. MMP-10 levels were low, whereas MMP-12 was not expressed in all cultures. Finally, MMP-13 amounts were highly influenced by substrate type, aza treatment, and culture duration.

TIMP-1 expression was abundant in all cultures, and the amounts were almost 10 -fold higher in 2D compared with 3D cultures. TIMP2 levels were also high in all cultures (TCP > rat collagen > human collagen) by Day 28. Although TIMP-3 was high in TCP cultures (loose or spheroids) at all time points, it was in intermediate levels in rat collagen gels and surprisingly was almost non-existent in human collagen gels. In general, aza showed inhibitory effect on TIMPs-1, 2 and 3 released within rat and human collagen gels. Taken together, these results suggest that $3 \mathrm{D}$ spheroid formation, 3D cultures, collagen source, and aza exposure directly influence levels of MMPs-1, 2, 3, 7, 8, 9, and 13 and TIMPs-1, 2, 3, and 4 releases (Figure 5).

\subsection{Global analysis of protein deposition and degradation}

Thus far, the levels of matrix proteins and secretome synthesized and released by cells were quantified and compared based on the culture conditions (aza presence, 2D vs. 3D culture, collagen species source, and culture duration). However, it would be more beneficial to gain a generalized mechanistic understanding of the relationship between matrix synthesis/deposition and factors contributing to that (role of matrix degrading and protecting enzymes). Thus, we pooled the normalized protein (total protein, elastin, GAGs, and LOX) amounts deposited as matrix or released into media, as quantified from various assays (Figure 2), from all culture conditions (2D and 3D, 0 and $10 \mu \mathrm{M}$ aza) tested in our study to identify respective time-dependent global trends. A modest decrease in matrix deposition of these proteins (Figure 6a) with a significant increase in their release into pooled media (Figure $6 \mathrm{~b}$ ), on a per cell basis, was noted over the 28-day culture period. We further investigated the underlying mechanisms for this paradox, that is, a time-dependent reduction in protein deposition 

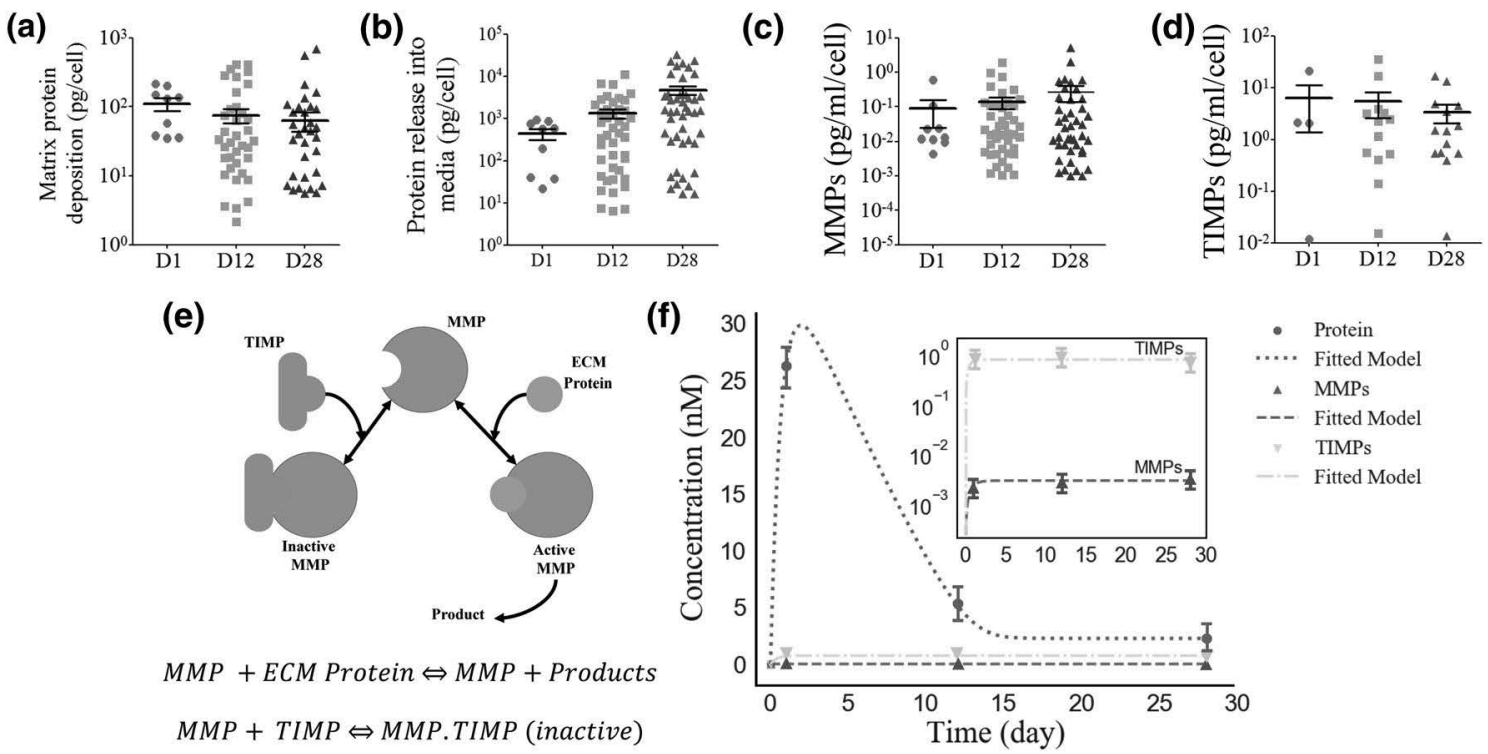

FIGURE 6 Scatter plots showing mean and standard error of pooled ECM (total protein, sGAG, HA, and elastin) proteins deposited as matrix (a) or released into pooled media (b), as quantified using various quantitative assays. Values were normalized to cell counts under respective culture conditions, as indicated. Similarly, the levels of MMPs (c) and TIMPs (d) released by cells into pooled media and quantified by immuno-beading assays. Values were normalized to cell count in respective cases. From these plots, a significant negative linear correlation $\left(R^{2}=.86\right)$ between matrix protein deposition and culture duration, a significant positive linear correlation $\left(R^{2}=.98\right)$ between protein release into pooled media and culture duration, a significant positive linear correlation $\left(R^{2}=.8\right)$ between MMPs released into pooled media and duration of culture, and a significant downregulation $\left(R^{2}=.8\right)$ of TIMPs release over the culture duration were noted. Schematic representation of the proposed competitive inhibition mechanism (Alberts, 2004). (e) TIMPs block the binding site of MMPs and prevent it from binding with substrate (matrix protein). (f) Equations (1)-(3) were fitted to the cumulative total protein deposition at each time point (experimental data pooled from across the cases) and the resulting curve fitting of the model. Symbols represent the mean \pm standard error of experimental data whereas dotted lines are the fitted models [Colour figure can be viewed at wileyonlinelibrary.com]

as matrix despite their accumulation in pooled media (Figure 6a vs. Figure $6 \mathrm{~b})$. It turns out that the levels of matrix degrading enzymes (i.e., MMPs) increased significantly with time (Figure 6c), whereas the availability of matrix-protecting enzymes (i.e., TIMPs) decreased in these cultures (Figure 6d). The two groups of enzymes in this system (MMPs and TIMPs) were produced by cells, and although MMPs degrade matrix proteins, TIMPs supposedly inhibit them from degrading matrix proteins.

As shown in Figure 6e, we assumed that (a) the production rate of enzymes by cells is constant over time; (b) binding of MMPs to TIMPs is reversible, as is binding of matrix protein molecules to MMPs; (c) TIMPs and matrix protein substrate are homologous in binding affinity and sites, and therefore compete to bind to free MMPs, leading to competitive inhibition; (d) either TIMP or matrix protein substrate could bind effectively to MMPs, but not both together; and (e) because matrix protein amounts are significantly higher than that TIMPs (Figure 6), they might easily overcome the competition with TIMPs. Equations 1 and 2 show the time-dependent concentration changes in MMPs and TIMPs over the 28-day culture period:

$$
\begin{aligned}
& \frac{d E_{M M P s}}{d t}=\alpha_{M M P s}-\beta_{M M P s} E_{M M P s}, \\
& \frac{d E_{T I M P s}}{d t}=\alpha_{T I M P s}-\beta_{T I M P s} E_{T I M P s},
\end{aligned}
$$

where $E_{\mathrm{MMPs}}$ and $E_{\mathrm{TIMPs}}$ are the concentrations of MMPs and TIMPs in $\mathrm{nM}$ respectively, $t$ is the time in days, $\alpha_{\mathrm{MMPs}}$ and $\alpha_{\mathrm{TIMPs}}$ are production rates $\left(\mathrm{nM} \cdot \mathrm{day}^{-1}\right.$ ), and $\beta_{\mathrm{MMPs}}$ and $\beta_{\text {TIMPs }}$ are the decay rate coefficients of MMPs and TIMPs respectively $\left(\right.$ day $\left.^{-1}\right)$. MMPs, released in an inactive form by eukaryotic cells, are usually activated and deactivated by other MMPs and TIMPs respectively (e.g., MMPs 2 or 9 binds to TIMP-1 in 1:1 stoichiometry).

To model the protein concentration in this system, we used the competitive inhibition form of Michaelis-Menten equation to estimate the degradation rate of protein:

$$
\frac{d p}{d t}=\alpha_{\mathrm{p}}-\frac{k_{\mathrm{cat}} E_{\mathrm{MMPs}} p}{\kappa_{\mathrm{m}}\left(1+\frac{\left[E_{\mathrm{TIMP}}\right]}{k_{\mathrm{TIMP}}}\right)+p},
$$

where $p$ is the protein concentration, $\alpha_{\mathrm{p}}$ is the protein production rate $\left(\mathrm{nM} \cdot \mathrm{day}^{-1}\right), k_{\mathrm{cat}}$ is the turnover number $\left(\mathrm{day}^{-1}\right), K_{\mathrm{m}}$ is the MichaelisMenten constant (nM), and $k_{\text {TIMPs }}$ is the binding constant (nM) of inhibitive enzyme (i.e., TIMPs). Again, we have assumed that the average cellular production rate of protein, and other parameters in Equation 3 , is constant over the 28 days of experiment. It should be noted that such measured matrix protein amounts also include the intracellular proteins in respective cultures at these time points.

Experimental data from three time points, that is, Days 1, 12, and 28 , were used to predict the protein concentration at intermediate 
time points. All simulations and optimization methods have been done in Python using the numpy, Imfit, and scipy libraries and leastsq as a fitting method. Curve-fitting results (Table 1 and Figure 6f) suggest an excellent match between the fitted model and the experimental data. The fitted curves for MMPs, TIMPs, and protein concentrations exhibited $R^{2} \geq 0.96$ indicating the success of this model in describing the experimental data.

\section{I DISCUSSION}

Recently, MSCs have emerged as a primary candidate for cell-based therapies, with around hundreds of clinical trials already conducted to test their suitability in treating various ailments, including cardiovascular diseases ( $14 \%$ of the total trials; Squillaro, Peluso, \& Galderisi, 2016). The immense clinical potential of MSCs has been attributed to their differentiability, immune-suppression, anti-apoptotic and anti-inflammatory roles, trophic and migration abilities to the injured/diseased tissues, and their ability to secrete multiple paracrine factors essential for tissue regeneration (Murphy, Moncivais, \& Caplan, 2013). These soluble paracrine factors serve as crucial mitogenic, proangiogenic, anti-apoptotic, immunomodulatory, anti-scarring, chemoattractive, and supportive factors during tissue regeneration (Meirelles, Fontes, Covas, \& Caplan, 2009). Besides these cytokines, MSCs also secrete various ECM proteins, proteases (MMPs), and their inhibitors (TIMPS) that play crucial role in matrix rebuilding and remodelling (Williams \& Hare, 2011). Thus, in recent years, there has been an immense interest to unveil the vast repertoire of MSC secretome, their mechanisms to induce tissue repair and regeneration, and approaches to modulate such outcomes.

MSC secretome exerts both positive and negative effects on cardiac regeneration. For instance, eotaxins 1, 2, and 3 (CCL11, CCL24, and CCL26, respectively) are involved in vascular diseases and endothelial dysfunction; MCP-1 and IL-1, 6, and 8 are pro-inflammatory; MMPs contribute to matrix degradation, whereas TGF- $\beta$ could lead to cardiac fibrosis (Williams \& Hare, 2011). Thus, it is important to not only promote beneficial paracrine factors release by MSCs but also explore mechanisms to minimize the release of inhibitory factors for better tissue regeneration outcomes. MSC secretome is also responsive to the local micro-environment cues; that is, inflammatory cytokines stimulate MSCs to secrete higher amounts of growth factors (Ma et al., 2014). Extrinsic techniques such as physiological preconditioning, gene manipulation, molecular and pharmaceutical preconditioning, cell-cell interactions, and physical pre-conditioning are needed to improve MSC survival post-transplantation and achieve controlled, predictable, and durable MSC secretome (Ranganath et al., 2012).

Spheroid formation is important as MSCs within spheroids preserve phenotype and innate properties and lead to enhanced cell survival, differentiation, and regenerative potential, compared with loose MSCs (Sart, Tsai, Li, \& Ma, 2013). Cells in spheroids coordinate with each other and with the released secretome to result in higher cellcell and cell-ECM interactions. In addition, cells also experience 
gradients of physical tension, cytokines, nutrients, and oxygen tension (e.g., mild hypoxia in the interior), and all these factors are hypothesized to precondition MSCs for enhanced secretion of pro-angiogenic and anti-inflammatory factors and ECM molecules (Sart et al., 2013). Specifically, the hypoxic core of MSC spheroids upregulates hypoxiaassociated genes, which are principally regulated by hypoxia inducible factor (HIF) master transcription factors (Cesarz \& Tamama, 2016; Sart et al., 2013). In hypoxic conditions, HIF-1a and HIF-2a exert crucial roles in MSC stemness and paracrine secretion, and the protein expression of both these transcription factors is observed in MSC spheroids (Cesarz \& Tamama, 2016; Sart et al., 2013). Thus, the hypoxic environment within MSC spheroids enhance pro-angiogenic growth factors (e.g., VEGF and HGF), upregulates anti-inflammatory molecules (e.g., PGE2, TSG-6), and confers higher differentiation and matrix synthesis ability of MSC spheroids, when compared with their loose counterparts (Bartosh et al., 2010; Cesarz \& Tamama, 2016; Sart et al., 2013). Apart from HIF, anti-apoptotic molecules such as Bcl-2 are upregulated in MSC spheroids that provide higher cell survival benefits post-transplantation and higher regeneration ability to MSC spheroids (Bartosh et al., 2010; Cesarz \& Tamama, 2016; Sart et al., 2013). In this context, our prior study examined time-dependent cardiomyogenic evolution from human BM-MSCs when MSC spheroids were cultured within $2 \mathrm{mg} / \mathrm{ml}$ (rat tail-derived) collagen hydrogel and treated with $10 \mu \mathrm{M}$ aza for 28 days (Joshi et al., 2018). Delivering such MSC-loaded hydrogels to the infarct myocardium will influence cardiac regeneration because they (a) provide immediate microenvironment for the transplanted cells and other newly infiltrated endogenous progenitor cells to home in and improve their cell survival, (b) deliver regenerative paracrine cargo that will awaken endogenous regeneration capability, and (c) replace the native lost cardiomyocytes with cardiomyocyte-like cells. However, it was not clear till now how this $3 \mathrm{D}$ milieu modulates the time-dependent secretome release and ECM deposition by the BM-MSC spheroids relevant for cardiac tissue regeneration.

Two-way ANOVA analysis suggested that culture condition (scaffold type and aza treatment, $p<.0001)$, culture duration $(p=.0004)$, and the interaction of these two factors $(p<.0001)$ exerted significant roles on cell proliferation. In addition, significant effects of culture condition $(p<.0001)$ and culture duration $(p=.002)$ on cell viability were noted, with rat collagen gels supporting significantly higher viability compared with human collagen gels at each culture duration, in the presence or absence of aza ( $p<.001$ for all cases). Taken together, results indicate benefits of rat collagen gels for cell proliferation and viability compared with human collagen gels. Normalized total protein, elastin, LOX, HA, and SGAG amounts, either deposited as matrix or released into pooled media, were significantly influenced by culture conditions, duration, and interaction of these two factors $(p<.0001$, for all). For reasons beyond the scope of this work, human collagen appeared to promote significantly higher release of most of these proteins compared with 2D cultures or rat collagen, whereas aza suppressed the amounts of these proteins in most cases.

On a per cell basis, the matrix amounts in 2D MSC spheroid cultures (Figure $2 \mathrm{~d}$ ) noted in our study were significantly higher than that reported in 2D cultures of loose MSCs. In this study, the normalized released amounts of total protein, sGAG, HA, and elastin on 2D substrates by Day 12 were $10^{6}, 3,500,60$, and $2,700 \mathrm{pg}$, respectively, and by Day 28 were $35 \times 10^{4}, 2,800,52$, and $3,600 \mathrm{pg}$, respectively. Amable, Teixeira, Carias, Granjeiro, and Borojevic (2014) reported that control BM-MSCs in 2D cultures released $<5$ pg of collagen I, $<50 \mathrm{pg}$ of collagen II, $<1,250 \mathrm{pg}$ of collagen III, $\sim 120 \mathrm{pg}$ of elastin, $\sim 300 \mathrm{pg}$ of heparin sulfate, $<3 \mathrm{pg}$ of decorin, $<0.5 \mathrm{pg}$ of aggrecan, and $4 \mathrm{pg}$ of laminin, per million cells per day. These amounts are much lower compared with the matrix protein levels noted in our study in 2D cultures (Figure 2), which could be attributed to differences in loose (in their study) versus spheroid cultures here.

Although cell proliferation and survival were compromised in 3D human collagen gels in our study, the normalized matrix amounts on a per cell basis were significantly higher than in 2D TCP or 3D rat collagen gels, even in aza-free conditions. Similar trends were noted for collagen III, laminin, fibrillin, NOS2 (iNOS), and NOS3 (eNOS) levels in human collagen gel cultures. These native myocardial components contribute to provide specific micro-environment niche that influences the endogenous progenitor cell differentiation process (Bayomy, Bauer, Qiu, \& Liao, 2012). Thus, higher myocardial matrix components deposition by BM-MSC spheroids, within 3D collagen gels and specifically within human collagen, infer their higher potential in recovering lost tissue following MI. Aza exposure reduced the expression of most of the myocardial proteins within rat and human collagen gels that could boost its application in the treatment of cardiac fibrosis, as DNA hypermethylation (for which aza is normally used) has been associated with many forms of cardiac fibrosis (Neary, Watson, \& Baugh, 2015). Thus, 3D collagen gels, specifically from human collagen, promoted synthesis of cardiac matrix components; however, reduced matrix synthesis with aza may refer to its anti-fibrotic role.

We noted that release of chemokines (IL-6, MCP-1, MCP-3, and GRO- $a$ ) and growth factors (FGF-2 and VEGF- $a$ ) reduced with spheroid formation and collagen cultures; human collagen and aza lowered chemokines release but promoted higher iNOS expression, a potent immunomodulatory factor. Because higher levels of chemokines in serum was detected in patients with heart diseases (Dusi, Ghidoni, Ravera, De Ferrari, \& Calvillo, 2016), the reduced levels of chemokines noted in our study could be beneficial in limiting the tissue inflammation of an inflamed myocardial micro-environment. Thus, collagen cultures and specifically human collagen led to reduced expression of chemokines and pro-inflammatory cytokines but caused concomitant higher expression of immunomodulatory factor; such tuned MSC profile could be beneficial when administered to inflamed myocardium.

Significant reduction in the deposition of elastin, sGAG, and HA proteins within 2D cultures, from Day 12 to Day 28, could be due to concomitant increases in MMP-2 release over this duration. Compared with 2D cultures, 3D collagen gels appear to have promoted higher secretion of cardiac tissue-relevant ECM proteins over the 28 days. We earlier reported that MSC spheroids within $2 \mathrm{mg} / \mathrm{ml}$ rat collagen gels (with $10 \mu \mathrm{m}$ aza) expressed early cardiomyocyte-like phenotype by Day 12 and late cardiomyocyte-like phenotype by Day 28 (Joshi et al., 2018). Thus, the differentiated cells within 3D collagen 
gels could be synthesizing and depositing more ECM proteins than their undifferentiated counterparts in 2D cultures. Although spheroid cultures in human collagen gels (with $10 \mu \mathrm{M}$ aza) promoted synthesis and release of sGAG, tropoelastin, HA, and LOX from Days 12 to 28, their deposition as matrix proteins was not proportionally realized. In general, 2D cultures promoted release of cardiac matrix proteins mostly until Day 12, whereas 3D collagen gels (rat or human) promoted release of such matrix proteins by differentiated cells, especially from Days 12 to 28 .

Collagen from different tissues undergo a series of unique posttranslational modifications that will govern the spatial organization, diameter, cross-linking, and D-spacing of the collagen fibrils (Fang \& Holl, 2013). In addition, the processing conditions, such as temperature, $\mathrm{pH}$, ionic strength, enzyme treatment, and the initial raw material, are known to influence collagen functional properties such as gelation rate, viscosity, and water retention capacity (Schmidt et al., 2016). Because collagen from rat-tail tendon is less cross-linked and more pure, dilute acid extraction is typically performed that retains the molecular domains of the collagen molecules. On the other hand, for collagens with higher cross-links, including human collagen (Vitrocol ${ }^{\circledR}$ ), enzymatic treatment is done, which cleaves telopeptides of collagen molecules (Kanta, 2015; Schmidt et al., 2016). Telopeptides principally participate in the intermolecular cross-links and impact the physical, biochemical, and mechanical properties of the hydrogels (Kanta, 2015; Schmidt et al., 2016). In addition, telopeptides are major antigenic determinants (Gorgieva \& Kokol, 2011); removal of telopeptides in human collagen eliminates the chances of immunogenic responses, if any, with the allogeneic collagen usage. Thus, differences in their extraction process could influence gelation time of human- and rat-derived collagens, possibly inducing variations in cell-collagen anchorage and adhesion and thereby proliferation. Although human collagen showed better biological properties (e.g., higher prosurvival factors), their physical characteristics such as higher gelation time might have impeded cell anchorage, survival, and proliferation compared with those in rat collagen. Thus, the unique 3D micro-environment governed by nanomorphological cues, fibril geometry, antigenic cues, and postprocessing states for rat and human collagens could have uniquely contributed to the observed MSC survival and functional responses.

We report here (for the first time) on the utility of a competitive inhibition form of Michaelis-Menten equation to estimate the degradation rate of a mixture of proteins in long-term stem cell cultures. Such mathematical treatment captures the dynamics of protein accumulation over time (deposition vs. degradation) and the underlying turnover mechanisms. Protein synthesis and degradation kinetics by bacteria and yeast cells have been widely reported using theoretical and experimental approaches (e.g., Li, Burkhardt, Gross, \& Weissman, 2014; Rothman, 2010), but such analysis in eukaryotic cell cultures is lacking, possibly due to the differences in time scales, complexity, and thermodynamics involved (Cohen et al., 2009). Prior studies reported on the association and dissociation constants of specific MMP/TIMP pairs; however, such data are not readily available for synthesis and decay rates of MMPs and TIMPs. MMP decay rate was reported to be $5 \times 10^{-5} / \mathrm{s}$ (Kim \& Friedman, 2010), which closely match the degradation rate $\left(\beta_{\mathrm{MMPs}}\right)$ derived in our simulations (Table 1). Because our model was trained on experimental observations, and there is a lack of pertinent literature on absolute values of production and decay rates of proteins and enzymes by mammalian cells (not their rate constants) or kinetics model parameters $\left(K_{\mathrm{m}}, K_{\text {cat }}\right.$, and $K_{\text {TIMPS }}$ ), our modelling results need to be verified independently by others. Although several important parameters were deduced by developing a mathematical model that represents the experiments, more work is needed to elucidate the variability in protein production dynamics in stem cell versus mature cardiomyocyte cultures and across various time segments.

In conclusion, our study reports for the first time on secretome release and ECM deposition and turnover in human BM-MSC spheroids within $3 \mathrm{D}$ collagen (rat and human), even in the presence of aza, over 28 days. We developed a mathematical model to understand the protein synthesis and deposition dynamics within these 28-day cultures and quantitatively assessed the critical role of MMP-TIMP nexus. Successful clinical application of autologous BM-MSCs for MI treatment is contingent not only on maximizing efficiency of cardiomyocyte differentiation and maturation but also on modulating cell secretome and MMPs-mediated ECM turnover. For effective clinical translation, further studies are needed to examine and compare the matrix synthesis and paracrine secretion ability of human MSCs, MSC spheroids, differentiated cardiomyocyte-like MSCs, and mature cardiomyocytes, over longer culture durations within both in vitro and in vivo conditions.

\section{ACKNOWLEDGEMENTS}

C. K. expresses his gratitude to the Cleveland State University Office of Research funds, and J. J. thanks the financial support from the Cellular and Molecular Medicine Specialization Fellowship and Dissertation Research Award from Cleveland State University.

\section{CONFLICTQFQNTEREST}

The authors have declared that there is no conflict of interest.

\section{REFERENCES}

Alberts, B. (2004). Essential cell biology. New York, NY: Garland Science Pub.

Altara, R., Manca, M., Sabra, R., Eid, A. A., Booz, G. W., \& Zouein, F. A. (2016). Temporal cardiac remodeling post-myocardial infarction: Dynamics and prognostic implications in personalized medicine. Heart Failure Reviews, 21(1), 25-47. https://doi.org/10.1007/s10741015-9513-8

Amable, P. R., Teixeira, M. V., Carias, R. B., Granjeiro, J. M., \& Borojevic, R. (2014). Protein synthesis and secretion in human mesenchymal cells derived from bone marrow, adipose tissue and Wharton's jelly. Stem Cell Research \& Therapy, 5(2), 53. https://doi.org/10.1186/scrt442 
Bartosh, T. J., Ylöstalo, J. H., Mohammadipoor, A., Bazhanov, N., Coble, K., Claypool, K., ... Prockop, D. J. (2010). Aggregation of human mesenchymal stromal cells (MSCs) into 3D spheroids enhances their antiinflammatory properties. Proceedings of the National Academy of Sciences of the United States of America, 107(31), 13724-13729. https://doi.org/10.1073/pnas.1008117107

Bayomy, A. F., Bauer, M., Qiu, Y., \& Liao, R. (2012). Regeneration in heart disease-Is ECM the key? Life Sciences, 91(17-18), 823-827. https:// doi.org/10.1016/j.lfs.2012.08.034

Bowers, S. L. K., Banerjee, I., \& Baudino, T. A. (2010). The extracellular matrix: At the center of it all. Journal of Molecular and Cellular Cardiology, 48(3), 474-482. https://doi.org/10.1016/j.yjmcc.2009.08.024

Cashman, T. J., Gouon-Evans, V., \& Costa, K. D. (2013). Mesenchymal stem cells for cardiac therapy: Practical challenges and potential mechanisms. Stem Cell Reviews, 9(3), 254-265. https://doi.org/10.1007/ s12015-012-9375-6

Cesarz, Z., \& Tamama, K. (2016). Spheroid culture of mesenchymal stem cells. Stem Cells International, 2016, 9176357.

Cohen, A. A., Kalisky, T., Mayo, A., Geva-Zatorsky, N., Danon, T., Issaeva, I., ... Alon, U. (2009). Protein dynamics in individual human cells: Experiment and theory. PLoS ONE, 4(4), e4901. https://doi.org/10.1371/ journal.pone.0004901

Dusi, V., Ghidoni, A., Ravera, A., De Ferrari, G. M., \& Calvillo, L. (2016). Chemokines and heart disease: A network connecting cardiovascular biology to immune and autonomic nervous systems. Mediators of Inflammation, 2016(2016), 5902947.

Fan, D., Takawale, A., Lee, J., \& Kassiri, Z. (2012). Cardiac fibroblasts, fibrosis and extracellular matrix remodeling in heart disease. Fibrogenesis \& Tissue Repair, 5(1), 15. https://doi.org/10.1186/1755-1536-5-15

Fang, M., \& Holl, M. M. (2013). Variation in type I collagen fibril nanomorphology: The significance and origin. BoneKEy reports, 2, 394.

Fomovsky, G. M., Thomopoulos, S., \& Holmes, J. W. (2010). Contribution of extracellular matrix to the mechanical properties of the heart. Journal of Molecular and Cellular Cardiology, 48(3), 490-496. https://doi. org/10.1016/j.yjmcc.2009.08.003

Förstermann, U., \& Sessa, W. C. (2011). Nitric oxide synthases: Regulation and function. European Heart Journal, 33(7), 829-837.

Frangogiannis, N. G. (2014). The inflammatory response in myocardial injury, repair, and remodelling. Nature Reviews. Cardiology, 11(5), 255-265. https://doi.org/10.1038/nrcardio.2014.28

Gishto, A., Farrell, K., \& Kothapalli, C. R. (2015). Tuning composition and architecture of biomimetic scaffolds for enhanced matrix synthesis by murine cardiomyocytes. Journal of Biomedical Materials Research. Part A, 103(2), 693-708. https://doi.org/10.1002/jbm.a.35217

Gorgieva, S., \& Kokol, V. (2011). Collagen- vs. gelatine-based biomaterials and their biocompatibility: Review and perspectives. In Biomaterials applications for nanomedicine. London: IntechOpen.

Joshi, J., Mahajan, G., \& Kothapalli, C. R. (2018). Three-dimensional collagenous niche and azacytidine selectively promote time-dependent cardiomyogenesis from human bone marrow-derived MSC spheroids. Biotechnology and Bioengineering, 115(8), 2013-2026. https://doi.org/ 10.1002/bit.26714

Kanta, J. (2015). Collagen matrix as a tool in studying fibroblastic cell behavior. Cell Adhesion \& Migration, 9(4), 308-316. https://doi.org/ 10.1080/19336918.2015.1005469

Kapelko, V. I. (2001). Extracellular matrix alterations in cardiomyopathy: The possible crucial role in the dilative form. Experimental and Clinical Cardiology, 6(1), 41-49.

Kielty, C. M., Sherratt, M. J., \& Shuttleworth, C. A. (2002). Elastic fibres. Journal of Cell Science, 115(14), 2817-2828.
Kim, Y., \& Friedman, A. (2010). Interaction of tumor with its microenvironment: A mathematical model. Bulletin of Mathematical Biology, 72, 1029-1068. https://doi.org/10.1007/s11538-009-9481-z

LeGrice, I., Pope, A., \& Smaill, B. (2005). The architecture of the heart: Myocyte organization and the cardiac extracellular matrix. In F. J. Villarreal (Ed.), Interstitial fibrosis in heart failure. Developments in cardiovascular medicine (Vol. 253) (pp. 3-21). New York, NY: Springer.

Li, G. W., Burkhardt, D., Gross, C., \& Weissman, J. S. (2014). Quantifying absolute protein synthesis rates reveals principles underlying allocation of cellular resources. Cell, 157(3), 624-635. https://doi.org/10.1016/j. cell.2014.02.033

Lockhart, M., Wirrig, E., Phelps, A., \& Wessels, A. (2011). Extracellular matrix and heart development. Birth Defects Research. Part A, Clinical and Molecular Teratology, 91(6), 535-550. https://doi.org/10.1002/ bdra. 20810

Ma, S., Xie, N., Li, W., Yuan, B., Shi, Y., \& Wang, Y. (2014). Immunobiology of mesenchymal stem cells. Cell Death and Differentiation, 21(2), 216-225. https://doi.org/10.1038/cdd.2013.158

Meirelles, L. S., Fontes, A. M., Covas, D. T., \& Caplan, A. I. (2009). Mechanisms involved in the therapeutic properties of mesenchymal stem cells. Cytokine \& Growth Factor Reviews, 20(5-6), 419-427. https:// doi.org/10.1016/j.cytogfr.2009.10.002

Murphy, M. B., Moncivais, K., \& Caplan, A. I. (2013). Mesenchymal stem cells: Environmentally responsive therapeutics for regenerative medicine. Experimental \& Molecular Medicine, 45(11), e54. https://doi.org/ 10.1038/emm.2013.94

Neary, R., Watson, C. J., \& Baugh, J. A. (2015). Epigenetics and the overhealing wound: The role of DNA methylation in fibrosis. Fibrogenesis \& Tissue Repair, 8(1), 18. https://doi.org/10.1186/ s13069-015-0035-8

Palamakumbura, A. H., \& Trackman, P. C. (2002). A fluorometric assay for detection of lysyl oxidase enzyme activity in biological samples. Analytical Biochemistry, 300(2), 245-251. https://doi.org/10.1006/ abio.2001.5464

Pelouch, V., Dixon, I. M., Golfman, L., Beamish, R. E., \& Dhalla, N. S. (1993). Role of extracellular matrix proteins in heart function. Molecular and Cellular Biochemistry, 129(2), 101-120. https://doi.org/10.1007/ BF00926359

Ranganath, S. H., Levy, O., Inamdar, M. S., \& Karp, J. M. (2012). Harnessing the mesenchymal stem cell secretome for the treatment of cardiovascular disease. Cell Stem Cell, 10(3), 244-258. https://doi.org/10.1016/ j.stem.2012.02.005

Rienks, M., Papageorgiou, A. P., Frangogiannis, N. G., \& Heymans, S. (2014). Myocardial extracellular matrix. Circulation Research, 114(5), 872-888. https://doi.org/10.1161/CIRCRESAHA.114.302533

Rothman, S. (2010). How is the balance between protein synthesis and degradation achieved? Theoretical Biology \& Medical Modelling, 7, 25. https://doi.org/10.1186/1742-4682-7-25

Sart, S., Tsai, A. C., Li, Y., \& Ma, T. (2013). Three-dimensional aggregates of mesenchymal stem cells: Cellular mechanisms, biological properties, and applications. Tissue Engineering. Part B, Reviews, 20(5), 365-380. https://doi.org/10.1089/ten.TEB.2013.0537

Schmidt, M. M., Dornelles, R. C. P., Mello, R. O., Kubota, E. H., Mazutti, M. A., Kempka, A. P., \& Demiate, I. M. (2016). Collagen extraction process. International Food Research Journal, 23(3), 913-922.

Simmers, P., Gishto, A., Vyavahare, N., \& Kothapalli, C. R. (2015). Nitric oxide stimulates matrix synthesis and deposition by adult human aortic smooth muscle cells within three-dimensional cocultures. Tissue Engineering. Part A, 21(7-8), 1455-1470. https://doi.org/10.1089/ten. tea.2014.0363 
Spinale, F. G. (2007). Myocardial matrix remodeling and the matrix metalloproteinases: Influence on cardiac form and function. Physiological Reviews, 87(4), 1285-1342. https://doi.org/10.1152/physrev.00012. 2007

Squillaro, T., Peluso, G., \& Galderisi, U. (2016). Clinical trials with mesenchymal stem cells: An update. Cell Transplantation, 25(5), 829-848. https://doi.org/10.3727/096368915X689622

Wang, L., Tran, I., Seshareddy, K., Weiss, M. L., \& Detamore, M. S. (2009). A comparison of human bone marrow-derived mesenchymal stem cells and human umbilical cord-derived mesenchymal stromal cells for cartilage tissue engineering. Tissue Engineering. Part A, 15(8), 2259-2266. https://doi.org/10.1089/ten.tea.2008.0393

Williams, A. R., \& Hare, J. M. (2011). Mesenchymal stem cells. Circulation Research, 109(8), 923-940. https://doi.org/10.1161/ CIRCRESAHA.111.243147

Zimmermann, W. H., Didié, M., Döker, S., Melnychenko, I., Naito, H., Rogge, C., ... Eschenhagen, T. (2006). Heart muscle engineering: an update on cardiac muscle replacement therapy. Cardiovascular Research, 71(3), 419-429. https://doi.org/10.1016/j.cardiores.2006.03.023

\section{SUPPORTING INFORMATION}

Additional supporting information may be found online in the Supporting Information section at the end of the article.

Data S1. The actual values of cytokines, chemokines, TGF-beta, MMPs and TIMPs released by cells cultured within tissue culture plastic (TCP), rat collagen gels, and human collagen gels.

How to cite this article: Joshi J, Abnavi MD, Kothapalli CR. Synthesis and secretome release by human bone marrow mesenchymal stem cell spheroids within three-dimensional collagen hydrogels: Integrating experiments and modelling. J Tissue Eng Regen Med. 2019;13:1923-1937. https://doi.org/ 10.1002/term.2943

Post-print standardized by MSL Academic Endeavors, the imprint of the Michael Schwartz Library at Cleveland State University, 